\title{
Quark mass dependence of light and heavy systems
}

\author{
F.-K. Guo*, M. Cleven*, C. Hanhart ${ }^{\dagger}$, F. J. Llanes-Estrada** and U.-G. Meißner ${ }^{\star}, \dagger$ \\ *Institut für Kernphysik and Jülich Center for Hadron Physics, Forschungszentrum Jülich, \\ D-52425 Jülich, Germany \\ ${ }^{\dagger}$ Institut für Kernphysik, Institute for Advanced Simulation and Jülich Center for Hadron Physics, \\ Forschungszentrum Jülich, D-52425 Jülich, Germany \\ ** Departamento de Física Teórica I, Universidad Complutense de Madrid, 28040 Madrid, Spain \\ ${ }^{*}$ Helmholtz-Institut für Strahlen- und Kernphysik and Bethe Center for Theoretical Physics, \\ Universität Bonn, D-53115 Bonn, Germany
}

\begin{abstract}
The pion mass dependence of the pion vector form factor is investigated using the Omnès representation. Our prediction agrees with later lattice results quite well. The nice agreement of the pion mass dependence of the scattering lengths of the charmed mesons and light pseudoscalar mesons suggests that the $D_{s 0}(2317)^{*}$ be a $D K$ bound state. Furthermore, it is argued that the kaon mass dependence of a kaonic bound state should be linear, and can be used as a criterion in determining the nature of such a bound state on lattice.
\end{abstract}

Keywords: Chiral extrapolation, pion form factor, hadronic molecule PACS: $11.30 . \mathrm{Rd}, 13.40 . \mathrm{Gp}, 13.75 . \mathrm{Lb}, 14.40 . \mathrm{Rt}$

Due to the non-perturbative nature of strong interaction at low energy, ab initio calculations are often performed on a lattice. However, nowadays, lattice calculations are normally performed using unphysical quark masses which are larger than the physical values. Therefore, an extrapolation to the physical point is necessary in order to get physical results on the lattice. This can be done using chiral perturbation theory (CHPT). In CHPT, the dependence of low-energy hadronic properties can be calculated up to a certain order in an expansion over light quark masses and low momenta. At leading order (LO), the light quark masses are related to the pion and kaon masses as

$$
M_{\pi}^{2}=2 B_{0} \bar{m}, \quad M_{K}^{2}=B_{0}\left(m_{s}+\bar{m}\right),
$$

where $\bar{m}=\left(m_{u}+m_{d}\right) / 2$, and $B_{0}$ is proportional to the quark condensate. From the above equation, one may express the light quark mass dependence as the pion or kaon mass dependence. In this talk, our recent study on the quark mass dependence of the pion vector form factor, the charmed meson-light meson scattering lengths, and the kaonic bound state mass will be briefly reviewed.

At low energy, the pion vector form factor can be expanded as

$$
F(t)=1+\frac{1}{3 !}\left\langle r^{2}\right\rangle t+\frac{1}{5 !}\left\langle r^{4}\right\rangle t^{2}+\mathscr{O}\left(t^{3}\right) .
$$

The relation between the quartic radius and curvature is $c_{V}^{\pi}=\frac{1}{5 !}\left\langle r^{4}\right\rangle$. The phase of the pion vector form factor is the same as the $\pi \pi$ scattering phase shift in the vectorisovector channel, $\delta_{11}$, below the first inelastic channel, which is known as Watson's theorem. Using dispersion relation and assuming the pion vector form factor does not have zeros, one may get the Omnès representation

$$
F(t)=\exp \left(\frac{\left\langle r^{2}\right\rangle}{6} t+\frac{t^{2}}{\pi} \int_{4 m_{\pi}^{2}}^{\infty} d s \frac{\delta_{11}(s)}{s^{2}(s-t-i \varepsilon)}\right),
$$

where we have made two subtractions in order to suppress the contribution from inelastic channels and from high energies. Taking the second derivative, we can get an expression for $c_{V}^{\pi}$ directly from Eq. (3):

$$
c_{V}^{\pi}=\frac{\left\langle r^{4}\right\rangle}{120}=\frac{1}{72}\left\langle r^{2}\right\rangle^{2}+\frac{1}{\pi} \int_{4 m_{\pi}^{2}}^{\infty} d s \frac{\delta_{11}(s)}{s^{3}} .
$$

We use two different methods to parameterize $\delta_{11}$. One is a simple Breit-Wigner (BW) method based on the assumption of $\rho$ meson dominance, and the other one is the inverse amplitude method (IAM) [1]. The parameters in these two methods ( $m_{\rho}$ and $g_{\rho \pi \pi}$ for the former and low-energy constants (LECs) for the latter) are fixed from fitting to the $\delta_{11}$ data. Then the pion vector form factor data in both the space-like and time-like regions can be described successfully using Eq. (3), for details see [2]. In order to do chiral extrapolation, one needs to know the pion mass dependence of $m_{\rho}$ and $g_{\rho \pi \pi}$ in the BW method. We proceed by assuming $g_{\rho \pi \pi}$ is $M_{\pi}$ independent, as indicated by unitarized CHPT [3], and using the $M_{\pi}$ dependence of the rho mass from lattice calculations [4]. In the IAM, the pion mass dependence follows from the interaction kernel obtained from the $\mathscr{O}\left(p^{4}\right)$ CHPT. From Eq. (4), we can predict the pion mass dependence of the quartic radius assuming the $M_{\pi}$ 

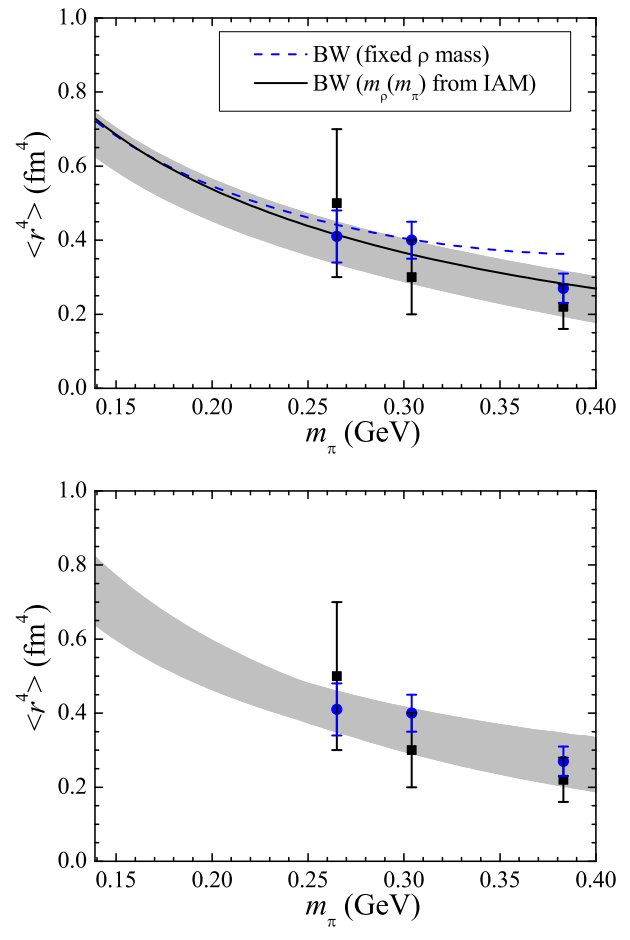

FIGURE 1. Predicted pion mass dependence of the quartic radius of the pion using the BW (top panel) and IAM (bottom panel) parametrization methods of the $\pi \pi$ phase shifts.

dependence of $\left\langle r^{2}\right\rangle$ is given by the $\mathscr{O}\left(p^{4}\right)$ CHPT. The prediction as shown in Fig. 1 agrees quite well with the lattice results reported in Ref. [5] after our prediction.

One may also study the quark mass dependence of heavy hadron properties based on chiral dynamics. For instance, the pion mass dependence of the scattering lengths of charmed mesons and light pseudoscalar mesons was investigated in [6]. The LO Lagrangian for the scattering is given by the Weinberg-Tomozawa term, and the next-to-leading order (NLO) Lagrangian contains several unknown LECs. After dropping terms which are not important, there are still three LECs, $h_{1}, h_{3}$ and $h_{5}$ (for details, see Ref. [7]), among which only $h_{1}$ can be determined from the mass difference between the $D_{s}^{+}$and $D^{+}$. To fix $h_{3}$ and $h_{5}$, one has to require $h_{5}$ to be of natural size, which is essential for an effective field theory to be convergent. Then for a given $h_{5}$, one can fix a value for $h_{3}$ assuming the $D_{s 0}^{*}(2317)$ is a hadronic molecule generated through charmed meson-light meson interaction in the (strangeness, isospin) $=(1,0)$ channel. This was done by making coincide the mass of the $D_{s 0}^{*}(2317)$ with the pole in the unitarized amplitude $T(s)$ of the relevant channel. The unitarized $S$-wave projected amplitude is given by the following equation [8]

$$
T(s)=V(s)[1-G(s) V(s)]^{-1},
$$

where $V(s)$ and $G(s)$ are the interaction kernel obtained from the NLO Lagrangian and loop function matrix, respectively. The unitarization procedure is essential since the generation of a bound state is non-perturbative. Using the so-fixed parameters, one may then predict the scattering lengths for various (strangeness, isospin) channels, and also their pion mass dependence. The pion mass enters through either the quark mass term in the Lagrangian, or the pion-charmed meson loop, or the pionmass dependence of the masses of the involved mesons. The scattering lengths for the charmed meson-light meson interaction have not been measured yet and notably difficult to measure. Fortunately, there has been the first lattice calculation of some of them recently [9]. Our predictions agree with the available lattice data well, see [6]. The comparison with the lattice data for the isospin-3/2 $D \pi$ channel is shown in the upper panel of Fig. 2, where the dashed line denotes the LO result without unitarization and the band denotes the unitarized NLO result. One sees that unitarization is necessary to render the prediction to be consistent with the lattice data. The nice agree-
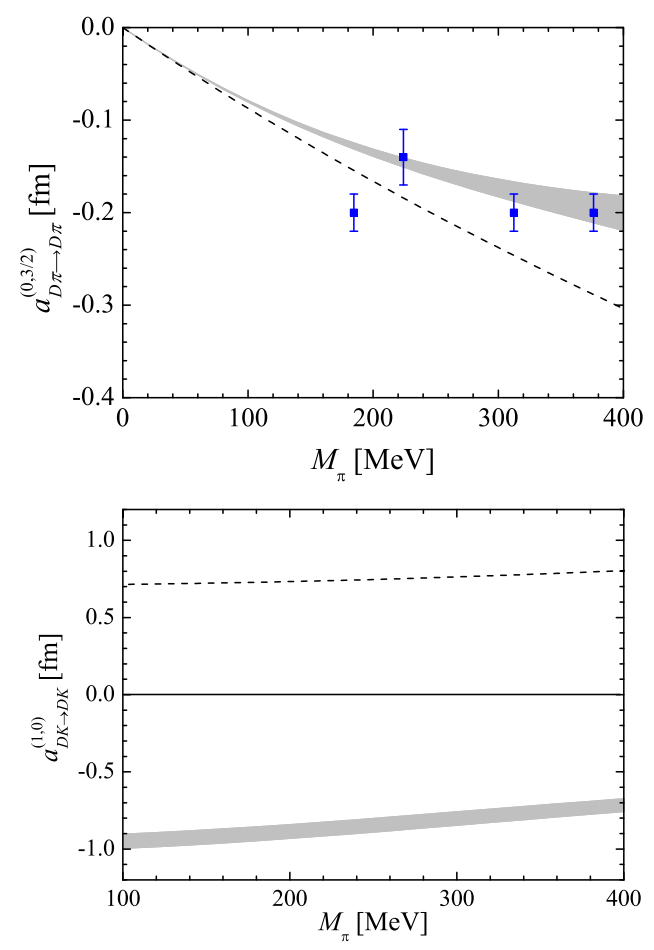

FIGURE 2. Predicted pion mass dependence of the charmed meson-light meson $S$-wave scattering lengths. 
ment, on the other hand, supports the assumption that the $D_{s 0}^{*}(2317)$ is a hadronic molecule whose main component is $D K$. The $D K$ scattering length for the $D_{s 0}^{*}(2317)$ channel is shown in the lower panel of Fig. 2. One can see unitarization changes the sign of the scattering length, and this may be expected as an $S$-wave bound state which mainly couples to the $D K$ appears below the $D K$ threshold.

The $D_{s 0}^{*}(2317)$ is an example of kaonic bound states. Other candidates include the $\Lambda(1405)$ as a $N \bar{K}$ bound state, the $f_{0}(980)$ as a $K \bar{K}$ bound state, the $D_{s 1}(2460)$ as a $D^{*} K$ bound state, and so on. Although most current lattice simulations are performed with physical strange quark mass, we have shown that varying the kaon mass on the lattice can provide very useful information for the nature of kaonic loosely bound states [10]. In general, the mass of a kaonic loosely bound state can be written as $M=M_{K}+M_{h}-\varepsilon$, where $M_{h}$ is the mass of the other hadron, and $\varepsilon \ll M_{K}$ is the binding energy. Although both $M_{h}$ and $\varepsilon$ have some kaon mass dependence, it is expected to be a lot weaker than that of the kaon itself. Thus, the leading kaon mass dependence of a kaonhadron bound state is linear, and the slope is unity (for the $f_{0}(980)$, the slope would be about two). Hence, as for the $D K$ bound state discussed here, we expect that the mass is linear in the kaon mass, and the slope is approximately one. Our explicit calculations as shown in the upper panel of Fig. 3, where the dashed line represents the $D K$ threshold, confirm this expectation. Were the $D_{s 0}^{*}(2317)$ a $c \bar{s}$ meson or a tetraquark, the kaon mass dependence would be quadratic. Also shown in Fig. 3 is the kaon mass dependence of the binding energy $\varepsilon_{D_{s 0}^{*}(2317)}=M_{D}+M_{K}-M_{D_{s 0}^{*}(2317)}$.

In summary, we have shown that using Omnès representation, the pion mass dependence of the quartic radius of the pion can be predicted successfully. The nice agreement of the predicted charmed meson-light meson $S$-wave scattering lengths with the lattice results supports the assumption of the $D_{s 0}^{*}(2317)$ being a hadronic molecule. As a specific example of kaonic bound states, it is shown the kaon mass dependence of the $D_{s 0}^{*}(2317)$ is linear, and the slope is about unity. One can learn much about the nature of hadrons from studying the light quark mass dependence of various hadronic properties.

\section{ACKNOWLEDGMENTS}

This work is partially supported by the Helmholtz Association through funds provided to the Virtual Institute "Spin and strong QCD" (VH-VI-231), by the DFG (TR 16, "Subnuclear Structure of Matter"), the European Community-Research Infrastructure Integrating Activity "Study of Strongly Interacting Matter" (acronym
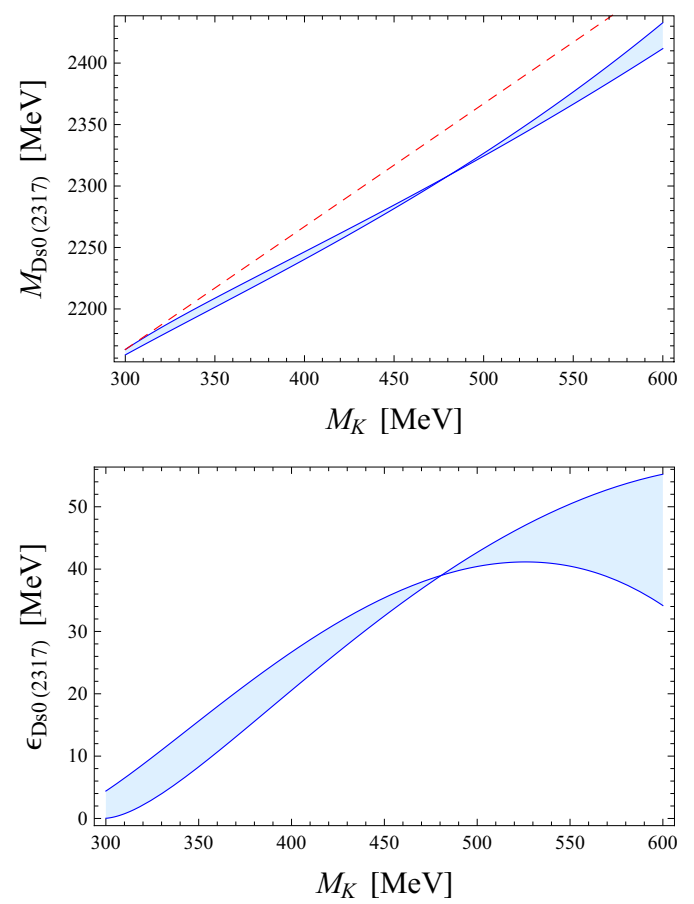

FIGURE 3. The kaon mass dependence of the mass of the $D_{s 0}^{*}(2317)$ (top panel) and the binding energy (bottom panel).

HadronPhysics2, Grant Agreement n. 227431) under the FP7 of the EU, and the BMBF (grant 06BN9006), and by grants FPA 2008-00592, FIS2008-01323 (Spain).

\section{REFERENCES}

1. A. Dobado, M. J. Herrero, and T. N. Truong, Phys. Lett. B235, 134 (1990).

2. F.-K. Guo, C. Hanhart, F. J. Llanes-Estrada, and U.-G. Meißner, Phys. Lett. B678, 90-96 (2009).

3. C. Hanhart, J. R. Pelaez, and G. Rios, Phys. Rev. Lett. 100, 152001 (2008).

4. M. Gockeler, et al., PoS LATTICE2008, 136 (2008).

5. R. Frezzotti, V. Lubicz, and S. Simula, Phys. Rev. D79, 074506 (2009).

6. F.-K. Guo, C. Hanhart, and U.-G. Meißner, Eur. Phys. J. A40, 171-179 (2009).

7. F.-K. Guo, C. Hanhart, S. Krewald, and U.-G. Meißner, Phys. Lett. B666, 251-255 (2008), 0806.3374.

8. J. A. Oller, and U. G. Meißner, Phys. Lett. B500, 263-272 (2001).

9. L. Liu, H.-W. Lin, and K. Orginos, PoS LATTICE2008, 112 (2008).

10. M. Cleven, F.-K. Guo, C. Hanhart, and U.-G. Meißner (2010), arXiv:1009.3804 [hep-ph]. 
Copyright of AIP Conference Proceedings is the property of American Institute of Physics and its content may not be copied or emailed to multiple sites or posted to a listserv without the copyright holder's express written permission. However, users may print, download, or email articles for individual use. 